\title{
reci@muc
}

Revista científica de investigación actualización del mundo de las ciencias

Carlos Bruno Guerrero Cedeño a; César Antonio Moran Vargas ${ }^{\text {b }}$; Gabriela Patricia Bajaña Aguilar ${ }^{c}$

Importancia de la capnografía durante el monitoreo anestésico

Importance of capnography during monitoring anesthetic

Revista Científica de Investigación actualización del mundo de las Ciencias. Vol. 3 núm., 2, septiembre, ISSN: 2588-0748, 2018, pp. 688-711

DOI: $10.26820 / \mathrm{reciamuc/3.(2).septiembre.2019.688-711}$

URL: http://reciamuc.com/index.php/RECIAMUC/article/view/362

Código UNESCO: 3205 Medicina Interna

Tipo de Investigación: Artículo de Revisión

(C) RECIAMUC; Editorial Saberes del Conocimiento, 2019

Recibido: 21/02/2019

Aceptado: 10/03/2019

Publicado: 01/09/2019

Correspondencia: drcarlosguerrero@ hotmail.es

a. Médico; Investigador Independiente; Guayaquil, Ecuador; drcarlosguerrero@ hotmail.es

b. Médico; Investigador Independiente; Guayaquil, Ecuador; cesarmoranv248930@gmail.com

c. Médico; Investigador Independiente; Guayaquil, Ecuador; gbajanaa@gmail.com 


\section{Importancia de la Capnografía durante el monitoreo anestésico}

Vol. 3, núm. 2., (2019)

Carlos Bruno Guerrero Cedeño; César Antonio Moran Vargas; Gabriela Patricia Bajaña Aguilar

\section{RESUMEN}

Capnografía, se ha convertido en una parte integral de monitoreo de la anestesia y ayuda a prevenir eventos que amenazan la vida. Los monitores usan espectrografía de infrarrojos, espectrografía de masas, espectrografía de Raman, analizadores foto acústica o dispositivos colorimétricos para medir dióxido de carbono en los gases respiratorios y luego dar una lectura numérica (capnometría) y una forma de onda (Capnografía). Hay dos tipos de capnógrafos: la corriente principal y de corriente secundaria. El capnograma o forma de onda nos da información acerca de la inspiración y la espiración, así como la arterial para poner fin a las diferencias de dióxido de carbono de marea. Clínicamente dióxido de carbono respirado refleja cambios en el metabolismo, la circulación, la respiración, la vía aérea y el sistema de respiración. Por lo tanto, lacapnografía como herramienta de monitoreo ha llegado para quedarse, y un estudio detallado de las formas de onda y su interpretación se ha convertido en obligatoria.

Palabras clave: Capnografía; Capnómetro; Capnograma; Anestesia; Vigilancia. 


\title{
Alteraciones endocrinólogas y metabólicas de las enfermedades criticas \\ Vol. 3, núm. 2., (2019) \\ Carlos Bruno Guerrero Cedeño; César Antonio Moran Vargas; Gabriela Patricia Bajaña Aguilar
}

\begin{abstract}
Capnography has become an integral part of anesthesia monitoring and helps prevent lifethreatening events. The monitors use infrared spectrography, mass spectrography, Raman spectrography, acoustic photo analyzers or colorimetric devices to measure carbon dioxide in respiratory gases and then give a numerical reading (capnometry) and a waveform (Capnography). There are two types of capnographs: the main and secondary current. The capnogram or waveform gives us information about inspiration and expiration, as well as arterial to end the differences in tidal carbon dioxide. Clinically breathed carbon dioxide reflects changes in metabolism, circulation, respiration, airway and breathing system. Therefore, capnography as a monitoring tool has come to stay, and a detailed study of waveforms and their interpretation has become mandatory.
\end{abstract}

Key words: Capnography; Capnometer; Capnogram; Anesthesia; Surveillance. 


\section{Importancia de la Capnografía durante el monitoreo anestésico}

Vol. 3, núm. 2., (2019)

Carlos Bruno Guerrero Cedeño; César Antonio Moran Vargas; Gabriela Patricia Bajaña Aguilar

\section{Introducción.}

La capnografía, la medición de CO2 en los gases respiratorios se ha convertido en una parte integral de la monitorización de la anestesia. Luft desarrolló el principio de la capnografía en 1943 a partir del conocimiento que el $\mathrm{CO} 2$ es uno de los gases que absorbe infrarrojos (IR) de radiación de una longitud de onda particular. La precisión de análisis rápido CO2 IR en la determinación de la concentración de dióxido de carbono alveolar fue establecido por Collier y sus colegas y el valor de la muestra de marea final establecido por Ramwell (Adams, 1989). En 1978 Holanda fue el primer país en adoptar capnografía como un estándar de monitorización durante la anestesia.

La capnografía es útil para mucho más que la comprobación de la posición del tubo endotraqueal. Proporciona información acerca de la producción de CO2, la perfusión pulmonar, la ventilación alveolar, patrones respiratorios y la eliminación de CO 2desde el circuito de anestesia y ventilador. Por lo tanto, brinda un método rápido y fiable para detectar condiciones peligrosas para la vida tales como la mala posición de los tubos traqueales, insuficiencia respiratoria, insuficiencia circulatoria y circuitos respiratorios defectuosos. La Sociedad Americana de Anestesiología (ASA) cerrado reivindicaciones estudio mostró que 34\% de los eventos dañinos involucra al sistema respiratorio y propuso que el $93 \%$ de estos casos se podría haber evitado mediante el control con oximetría de pulso, capnografía o ambos.3Por esta razón, el ASA incluido capnografía en sus normas para el control básico. La Sociedad India de Anestesiólogos ha designado la capnografía como un estándar 'deseable' en sus "normas de control de anestesia recomendados para la India" a partir del 30 diciembre de 1999(Website of the Indian Society of Anaesthesiologists, 2000). 


\section{Alteraciones endocrinólogas y metabólicas de las enfermedades criticas \\ Vol. 3, núm. 2., (2019) \\ Carlos Bruno Guerrero Cedeño; César Antonio Moran Vargas; Gabriela Patricia Bajaña Aguilar}

\section{Terminología}

La capnografía es la representación gráfica de la concentración instantánea de CO2 en función del tiempo (Time Capnograma) o el volumen expirado (Capnograma volumen) durante un ciclo respiratorio.

Capnógrafo es la máquina que genera una forma de onda y el capnograma es la forma de onda real.

Capnometría es la medición y visualización numérica de inspiratoria máxima y concentraciones de $\mathrm{CO} 2$ espiratorio durante un ciclo respiratorio.

Capnómetro es el dispositivo que realiza la medición y muestra la lectura.

Sin embargo, el capnógrafo o capnograma, es mucho más preferible a un metro o incluso una pantalla digital rápida. En la práctica anestésica respiración a respiración de forma de onda se debe mostrar para permitir la vigilancia continua y análisis (Dorsch, 1999)

\section{Metodología.}

Para el desarrollo de este proceso investigativo, se plantea como metodología la encaminada hacia una orientación científica particular que se encuentra determinada por la necesidad de indagar en forma precisa y coherente una situación, en tal sentido (Dávila, 2015) define la metodología "como aquellos pasos previos que son seleccionados por el investigador para lograr resultados favorables que le ayuden a plantear nuevas ideas”. (p.66) 


\section{Importancia de la Capnografía durante el monitoreo anestésico}

Vol. 3, núm. 2., (2019)

Carlos Bruno Guerrero Cedeño; César Antonio Moran Vargas; Gabriela Patricia Bajaña Aguilar

Lo citado por el autor, lleva a entender que el desarrollo de la acción investigativa busca simplemente coordinar acciones enmarcadas en una revisión bibliográfica con el fin de complementar ideas previas relacionadas a la importancia de la capnografía en el monitoreo durante la anestesia a través de una revisión de literatura, para así finalmente elaborar un cuerpo de consideraciones generales que ayuden a ampliar el interés propuesto.

\section{Tipo de Investigación}

Dentro de toda práctica investigativa, se precisan acciones de carácter metodológico mediante las cuales, se logra conocer y proyectar los eventos posibles que la determinan, así como las características que hacen del acto científico un proceso interactivo ajustado a una realidad posible de ser interpretada. En este sentido, se puede decir, que la presente investigación corresponde al tipo documental, definido por Castro (2016), "se ocupa del estudio de problemas planteados a nivel teórico, la información requerida para abordarlos se encuentra básicamente en materiales impresos, audiovisuales y /o electrónicos”. (p.41).

En consideración a esta definición, la orientación metodológica permitió la oportunidad de cumplir con una serie de actividades inherentes a la revisión y lectura de diversos documentos donde se encontraron ideas explicitas relacionadas con los tópicos encargados de identificar a cada característica insertada en el estudio. Por lo tanto, se realizaron continuas interpretaciones con el claro propósito de revisar aquellas apreciaciones o investigaciones propuestas por diferentes investigadores relacionadas con el tema de interés, para luego dar la respectiva argumentación a los planteamientos, en función a las necesidades encontradas en la indagación. 


\section{Alteraciones endocrinólogas y metabólicas de las enfermedades criticas \\ Vol. 3, núm. 2., (2019) \\ Carlos Bruno Guerrero Cedeño; César Antonio Moran Vargas; Gabriela Patricia Bajaña Aguilar}

\section{Fuentes Documentales}

El análisis correspondiente a las características que predomina en el tema seleccionado, llevan a incluir diferentes fuentes documentales encargadas de darle el respectivo apoyo y en ese sentido cumplir con la valoración de los hechos a fin de generar nuevos criterios que sirven de referencia a otros procesos investigativos. Para (CASTRO, 2016) las fuentes documentales incorporadas en la investigación documental o bibliográfica, "representa la suma de materiales sistemáticos que son revisados en forma rigurosa y profunda para llegar a un análisis del fenómeno". (p.41). Por lo tanto, se procedió a cumplir con la realización de una lectura previa determinada para encontrar aquellos aspectos estrechamente vinculados con el tema, con el fin de explicar mediante un desarrollo las respectivas apreciaciones generales de importancia.

\section{Técnicas para la Recolección de la Información}

La conducción de la investigación para ser realizada en función a las particularidades que determinan a los estudios documentales, tiene como fin el desarrollo de un conjunto de acciones encargadas de llevar a la selección de técnicas estrechamente vinculadas con las características del estudio. En tal sentido, (Bolívar, 2015), refiere, que es "una técnica particular para aportar ayuda a los procedimientos de selección de las ideas primarias y secundarias”. (p. 71).

Por ello, se procedió a la utilización del subrayado, resúmenes, fichaje, como parte básica para la revisión y selección de los documentos que presentan el contenido teórico. Es decir, que mediante la aplicación de estas técnicas se pudo llegar a recoger informaciones en cuanto a la revisión bibliográfica de los diversos elementos encargados de orientar el proceso de investigación. Tal como lo expresa, (Bolívar, 2015) "las técnicas documentales proporcionan las herramientas 


\section{Importancia de la Capnografía durante el monitoreo anestésico}

Vol. 3, núm. 2., (2019)

Carlos Bruno Guerrero Cedeño; César Antonio Moran Vargas; Gabriela Patricia Bajaña Aguilar

esenciales y determinantes para responder a los objetivos formulados y llegar a resultados efectivos" (p. 58). Es decir, para responder con eficiencia a las necesidades investigativas, se introdujeron como técnica de recolección el método inductivo, que hizo posible llevar a cabo una valoración de los hechos de forma particular para llegar a la explicación desde una visión general.

Asimismo, se emplearon las técnicas de análisis de información para la realización de la investigación que fue ejecutada bajo la dinámica de aplicar diversos elementos encargados de determinar el camino a recorrer por el estudio, según, (Bolívar, 2015) las técnicas de procesamiento de datos en los estudios documentales "son las encargadas de ofrecer al investigador la visión o pasos que debe cumplir durante su ejercicio, cada una de ellas debe estar en correspondencia con el nivel a emplear" (p. 123). Esto indica, que para llevar a cabo el procesamiento de los datos obtenidos una vez aplicado las técnicas seleccionadas, tales como: fichas de resumen, textual, registros descriptivos entre otros, los mismos se deben ajustar al nivel que ha sido seleccionado.

\section{Resultados.}

Los métodos para medir los niveles de $\mathrm{CO} 2$ incluyen espectrografía de infrarrojos, espectrografía de Raman, espectrografía de masas, espectrografía foto acústica y análisis colorimétrico química.

El método de infrarrojos es el más ampliamente utilizado y más rentable. Los rayos infrarrojos se emiten por todos los objetos calientes y son absorbidos por gases no elementales (es decir, los compuestos por átomos diferentes), mientras que ciertos gases absorben longitudes de onda particulares que producen bandas de absorción en el espectro electromagnético IR. La 


\section{Alteraciones endocrinólogas y metabólicas de las enfermedades criticas}

Vol. 3, núm. 2., (2019)

Carlos Bruno Guerrero Cedeño; César Antonio Moran Vargas; Gabriela Patricia Bajaña Aguilar

intensidad de la radiación IR proyectada a través de una mezcla de gases que contiene CO2se ve disminuida por absorción; esto permite que el $\mathrm{CO} 2$ banda de absorción a ser identificado y es proporcional a la cantidad de $\mathrm{CO} 2$ en la mezcla.

Los rayos infrarrojos tienen una longitud de onda mayor que $1 \mathrm{~mm}$ y por lo tanto se encuentran más allá del espectro visible $(0.4-0.8 \mathrm{~mm})$; CO2 muestra una fuerte absorción en el IR lejos a 4,3 mm y por lo tanto se utiliza esta longitud de onda en el rango IR lejos (Kalenda, 2009)

La espectrografía Raman utiliza el principio de “dispersión Raman” para la medición de CO2. La muestra de gas es aspirada en la cámara de análisis, donde la muestra es iluminada por un haz de láser de argón monocromática de alta intensidad. La luz es absorbida por las moléculas, que son entonces excitado a estados de energía vibracional o rotativos inestables (dispersión Raman). Las señales de dispersión Raman (luz Raman) se miden a continuación. El espectro de líneas de dispersión Raman se puede utilizar para identificar todos los tipos de moléculas en la fase incluyendo gas $\mathrm{CO} 2$ y agentes inhalatorios. El espectrógrafo de masas separa gases y vapores de diferente peso molecular en función de su masa para cargar proporciones en un espectro. Mediante el análisis de espectro, la composición y la abundancia relativa de cada gas en una muestra se puede determinar. Se mide la concentración de los volúmenes por ciento y no la presión parcial. Los espectrómetros de masas son bastante caros y demasiado voluminoso para usar en la cabecera y rara vez se utilizan en la actualidad (Raemer \& Philip, 1990).

La foto acústica de medición de gas (PAS) se basa en el mismo principio que los analizadores de gas basado en IR convencionales: la capacidad de $\mathrm{CO} 2$, N2O y agentes anestésicos para absorber la luz IR. Sin embargo, difieren en técnicas de medición. Mientras espectrografía 


\section{Importancia de la Capnografía durante el monitoreo anestésico}

Vol. 3, núm. 2., (2019)

Carlos Bruno Guerrero Cedeño; César Antonio Moran Vargas; Gabriela Patricia Bajaña Aguilar infrarroja utiliza métodos ópticos, PAS utiliza una técnica acústica. Sin embargo, este método no ha ganado tanto renombre como espectrografía IR.

El método colorimétrico de la medición de $\mathrm{CO} 2$ emplea un indicador de espuma tratada químicamente contenida en una carcasa de plástico que funciona como un adaptador de codo tubo endotraqueal. El color del adaptador de espuma cambia cuando se expone a dióxido de carbono exhalado. La falta de una forma de onda limita su utility (Mollgaard, 2009).

\section{El Efecto de la presión atmosférica sobre el CO2}

Un cambio en la presión atmosférica influye directamente en la lectura de capnógrafos desde concentración de CO2 se mide como la presión parcial (efecto directo). Un aumento en la presión aumenta proporcionalmente el número de IR de absorción de CO2 moléculas y de ese modo aumenta la señal de CO2. Un aumento del $1 \%$ en la presión provoca un aumento relativo del $1 \%$ en la señal de CO2. Este efecto es eliminado por la calibración del capnógrafo con una presión conocida parcial del gas $\mathrm{CO} 2(\mathrm{mmHg}=\%$ en volumen $*$ presión atmosférica $)$ utilizando un gas de calibración disponible comercialmente.

Cambios en la presión también producen un efecto de segundo (alteración de las fuerzas intermoleculares) ejercida por moléculas de CO2, lo que altera la absorción IR. Un aumento en la presión por $1 \%$ resulta en un aumento relativo de la señal por 0,5 a $0,8 \%$, lo que puede producir un pequeño error. cambios máximos en la presión atmosférica causada por los cambios en el tiempo son del orden de $20 \mathrm{mmHg}$. Esto daría lugar a un cambio en la PCO2de menos de 0,5-0,8 mmHg. Por lo tanto, en el uso clínico de rutina, las correcciones para los cambios en la presión atmosférica son innecesarios. Aplicación de PEEP (presión espiratoria final positiva) aumenta la 


\section{Alteraciones endocrinólogas y metabólicas de las enfermedades criticas}

Vol. 3, núm. 2., (2019)

Carlos Bruno Guerrero Cedeño; César Antonio Moran Vargas; Gabriela Patricia Bajaña Aguilar

lectura de CO2. Un PEEP de $20 \mathrm{~cm} \mathrm{H} 2 \mathrm{O}$ aumenta la lectura de CO2en 1,5 mmHg. Algunas unidades miden la presión en el sensor y ajustan automáticamente la lectura de CO2 en consecuencia (Capnography, s.f.)

La influencia del vapor de agua

Los resultados erróneos ocurrirán si el agua que tiene una alta absorbancia IR entra en la célula. El vapor de agua es invariablemente presente en el aire espirado (a 37oC). Este se condensa a baja temperatura ambiente en las paredes del tubo de muestreo. Se requiere un sistema de separación de agua efectiva para uso continuo. Una trampa de agua se utiliza en los analizadores de la corriente lateral para eliminar el agua en forma de partículas antes de que pueda entrar en la célula de análisis. El diseño de la trampa se basa en las fuerzas gravitatorias para separar gotas de agua de la corriente de gas y la trampa se debe con frecuencia seca y la atención dirigida a prevenir la acumulación de agua.

La presencia de vapor de agua también afecta a la lectura ya que normalmente la temperatura de la paciente es $37 \mathrm{o} \mathrm{C}$, mientras que la de la célula instrumento es, digamos, $25^{\circ} \mathrm{C}$, es decir, una diferencia de $3 \mathrm{kPa}(23 \mathrm{mmHg})$ en $\mathrm{PH} 2 \mathrm{O}$ que resulta en una sobreestimación de PCO2 por 0,15\% (0.15kPa, 1,13 mmHg) (Adams, 1989).

\section{Fisiología básica de un capnograma}

Al final de la inspiración, suponiendo que no hay reinspiración, las vías respiratorias y los pulmones se llenan de CO2. El dióxido de carbono se difunde en los alvéolos y se equilibra con la sangre extrema capilar -alveolar $(\mathrm{PA} \mathrm{CO} 2=\mathrm{PCCo} 2=40 \mathrm{mmHg})$. La concentración real de CO2 


\section{Importancia de la Capnografía durante el monitoreo anestésico}

Vol. 3, núm. 2., (2019)

Carlos Bruno Guerrero Cedeño; César Antonio Moran Vargas; Gabriela Patricia Bajaña Aguilar

en los alvéolos se determina por la medida de la ventilación y la perfusión en los alvéolos (relación $\mathrm{V} / \mathrm{Q})$.

Los alvéolos con ventilación superior en relación con la perfusión (alta V / Q alvéolos) tienen menor CO2 en comparación con alvéolos con baja relación V / Q que tendrían mayor CO2. Como uno se mueve proximalmente en el tracto respiratorio, la concentración de CO2poco a poco cae a cero en algún momento. El volumen de gas exento de $\mathrm{CO} 2$ se denomina espacio muerto respiratorio y aquí no hay intercambio de oxígeno $(\mathrm{O} 2)$ y compañía entre los gases inspirados y la sangre. Como el paciente exhala, un sensor de $\mathrm{CO} 2$ en la boca detectará ninguna partícula de $\mathrm{CO} 2$ como gas inicial muestreada será el gas libre de CO2 desde el espacio muerto. Como exhalación continúa, la concentración de $\mathrm{CO} 2$ se eleva gradualmente y alcanza un pico como el CO2 gases ricos de los alvéolos se abren camino al punto de detecciónCO2 en la boca. Al final de la exhalación la concentración CO2 disminuye a cero (línea de base) como el paciente comienza la inhalación delos gases libres de CO2. El paso de CO2 de los alvéolos a la boca durante la exhalación y la inhalación de los gases libresde CO2 durante la inspiración da la forma característica para la curva de $\mathrm{CO} 2$, que es idéntico en todos los seres humanos con pulmones sanos. Cualquier desviación de esta forma debe ser investigado para determinar una causa fisiológica o patológica que produce la anormalidad (Capnography, s.f.)

\section{Las aplicaciones clínicas de la capnografía}

El CO2 se produce en el cuerpo por el metabolismo celular, transportado por el sistema circulatorio a los pulmones, excretados por los pulmones y se transportan por el sistema(Dorsch, 1999).respiración Por lo tanto, los cambios en CO2 respirado pueden reflejar alteraciones en el 


\section{Alteraciones endocrinólogas y metabólicas de las enfermedades criticas}

Vol. 3, núm. 2., (2019)

Carlos Bruno Guerrero Cedeño; César Antonio Moran Vargas; Gabriela Patricia Bajaña Aguilar

metabolismo, la circulación, la respiración, la vía aérea, o el sistema de respiración. final espiratorio presión parcial de CO2 normal oscila entre 35 y 45 mmHg (Fig.1 y 2).

Figura 1: dióxido de carbono al final de la marea baja, con una buena meseta alveolar puede ser el resultado de la hiperventilación o un aumento de la ventilación del espacio muerto.

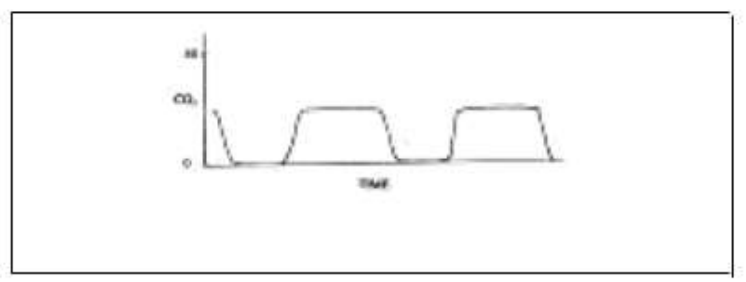

Figura 2: dióxido de carbono al final de la marea elevada con buena meseta alveolar puede ser causada por hipoventilación

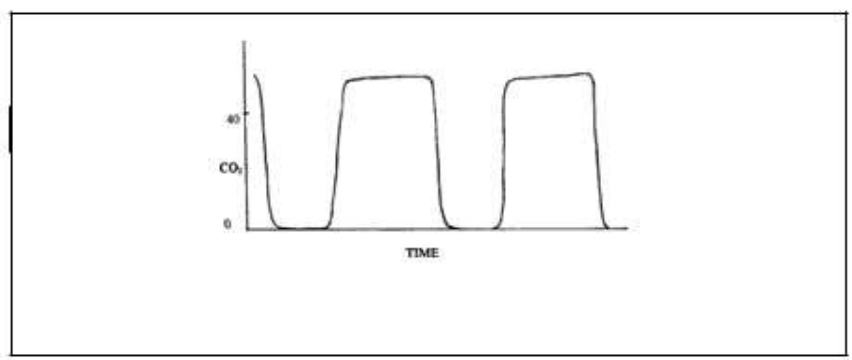

\section{Metabolismo}

Un aumento en la final de la espiración CO2es un indicador fiable de aumento del metabolismo sólo en pacientes ventilados mecánicamente. En pacientes con respiración espontánea, PET CO2 no puede aumentar como resultado de la hiperventilación causas metabólicas de los aumentos de $\mathrm{CO} 2$ espirado incluyen el aumento de temperatura, temblores, 


\section{Importancia de la Capnografía durante el monitoreo anestésico}

Vol. 3, núm. 2., (2019)

Carlos Bruno Guerrero Cedeño; César Antonio Moran Vargas; Gabriela Patricia Bajaña Aguilar

convulsiones, producción excesiva de catecolaminas, la administración de sangre o bicarbonato, la liberación de una pinza arterial o torniquete con reperfusión de áreas isquémicas (Fig.3), la glucosa en el fluido intravenosa, hiperalimentación parenteral, y CO2 usado para inflar la cavidad peritoneal durante la laparoscopia, la cavidad pleural durante toracoscopia o una articulación durante la artroscopia.

Figura 3: la liberación de un torniquete o desbloqueo de un vaso principal puede resultar en un aumento repentino de final de la espiración de dióxido de carbono que devuelve gradualmente a la normalidad.

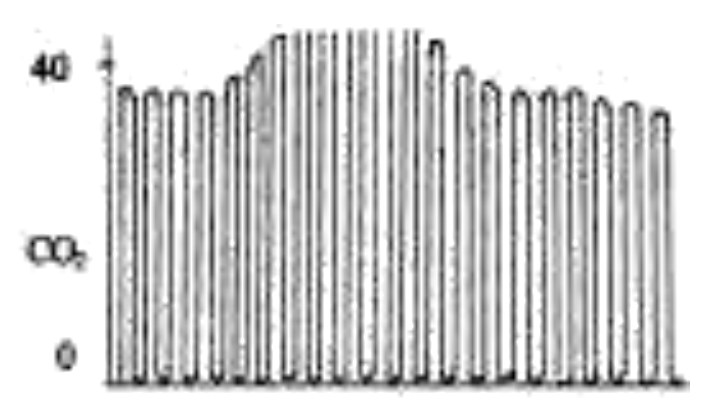

71sfe.

La hipertermia maligna es un estado hipermetabólico con un aumento masivo de producción de $\mathrm{CO} 2$. El aumento se produce al principio, antes de que el aumento de la temperatura. La detección temprana de este síndrome es una de las razones más importantes para el seguimiento de forma rutinaria $\mathrm{CO}$. Capnografía puede ser monitoreada para la eficacia del tratamiento.

La producción de $\mathrm{CO} 2$ cae con la temperatura disminuido, el aumento de la relajación muscular y aumento de la profundidad de la anestesia. 


\section{Alteraciones endocrinólogas y metabólicas de las enfermedades criticas \\ Vol. 3, núm. 2., (2019) \\ Carlos Bruno Guerrero Cedeño; César Antonio Moran Vargas; Gabriela Patricia Bajaña Aguilar}

\section{Circulación}

Una disminución en el final de la espiración CO2 se ve con una disminución en el gasto cardíaco si la ventilación se mantiene constante. La reducción del flujo de sangre a los pulmones también puede ser resultado de manipulaciones quirúrgicas de los vasos del corazón o torácicos, acuñamiento de un catéter en la arteria pulmonar, y la embolia pulmonar (trombo, tumor, grasa gas, ósea, o líquido amniótico).

Una disminución rápida de PET CO2en la ausencia de cambios en la presión arterial, la presión venosa central y el ritmo cardíaco indica una embolia de aire sin consecuencias hemodinámicas sistémicas. Sin embargo, como el tamaño de los aumentos de embolia de aire, una reducción de la producción se produce lo que disminuye aún más PET CO2 (Fig.4,5).

Figura 4: los eventos que causan una disminución exponencial en la final de la espiración de CO2incluir hipotensión repentina, paro circulatorio y la embolia pulmonar.

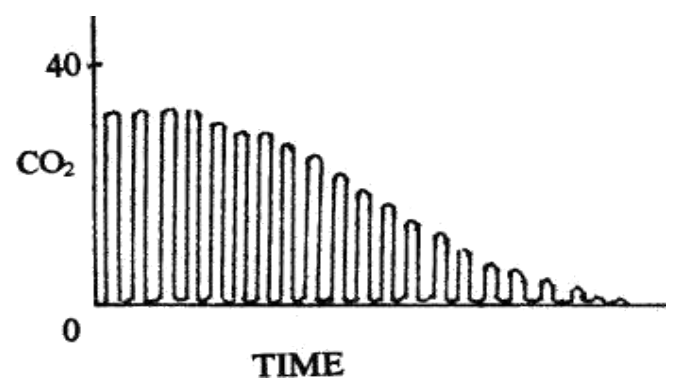




\section{Importancia de la Capnografía durante el monitoreo anestésico}

Vol. 3, núm. 2., (2019)

Carlos Bruno Guerrero Cedeño; César Antonio Moran Vargas; Gabriela Patricia Bajaña Aguilar

Figura 5: émbolo de aire pequeña

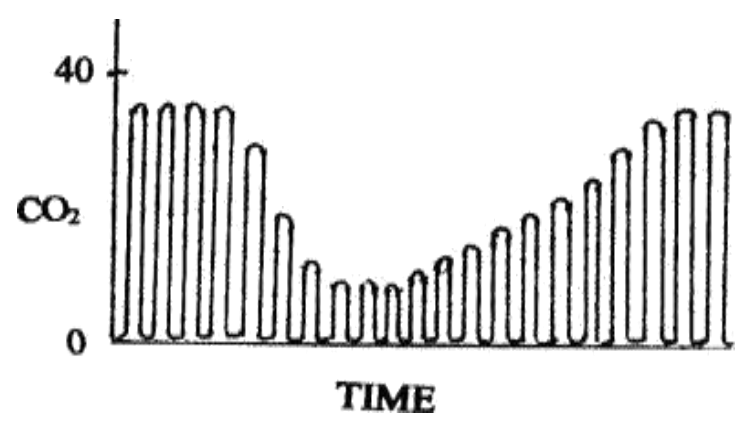

Durante la reanimación, exhalado de $\mathrm{CO}_{2}$ es una mejor guía a la presencia de circulación que el electrocardiograma (ECG), el pulso, o la presión arterial. La eficacia de las medidas de reanimación se puede medir por la capnografía. El capnograma no es susceptible a los artefactos mecánicos asociados con la compresión del pecho, Sin embargo, si se utiliza epinefrina dosis alta o bicarbonato, final de la espiración $\mathrm{CO}_{2}$ no es un buen indicador de las medidas de reanimación.

Un aumento repentino de $\mathrm{CO} 2$ al final de la marea durante la reanimación es un indicio temprano de que el gasto cardíaco espontáneo ha sido restaurada.

Las concentraciones de $\mathrm{CO} 2$ exhalado son útiles para determinar qué pacientes son susceptibles de ser reanimados con éxito. El paciente es más probable que ser resucitado si la concentración de CO2 exhalado es mayor que 10-15 mmHg.

La epinefrina inyectada por vía subcutánea se ha demostrado que aumenta el CO2 tidal final. Una posible explicación es que el gasto cardíaco se puede aumentar con CO2 adicional 


\section{Alteraciones endocrinólogas y metabólicas de las enfermedades criticas}

Vol. 3, núm. 2., (2019)

Carlos Bruno Guerrero Cedeño; César Antonio Moran Vargas; Gabriela Patricia Bajaña Aguilar

siendo transportado a los pulmones. La vasoconstricción periférica podría aumentar el volumen sanguíneo central y el flujo sanguíneo pulmonar.

Las oscilaciones cardiogénicas (Fig.6) aparecen como pequeños, regular, diente como jorobas al final de la fase espiratoria. Se cree que se debe a la contracción y relajación del corazón y los grandes vasos intratorácicos en los pulmones, forzando el aire dentro y fuera. Se ven generalmente a bajas tasas respiratorias y en los niños.

Figura 6: Las oscilaciones cardiogénico aparecen como dientes pequeños o jorobas regulares al final de la fase de espiración.

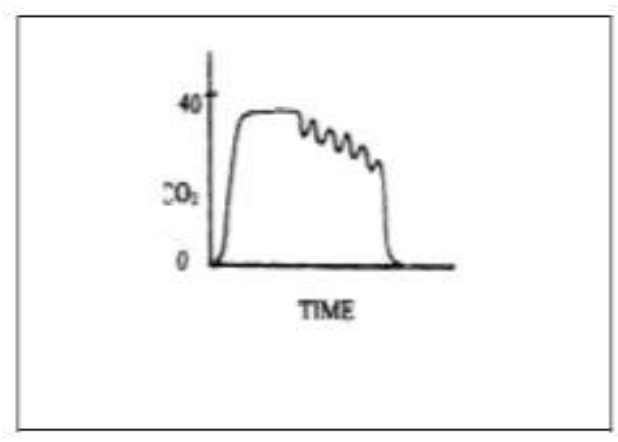

Respiración

El principal uso de la señal de capnografía en la anestesia es la verificación inmediata de la intubación traqueal fuera de toda duda por la presencia inmediata y continua de metabólica CO2 en el gas espirado. 


\section{Importancia de la Capnografía durante el monitoreo anestésico}

Vol. 3, núm. 2., (2019)

Carlos Bruno Guerrero Cedeño; César Antonio Moran Vargas; Gabriela Patricia Bajaña Aguilar

La detección de $\mathrm{CO} 2$ de la colocación del esófago tiene algunos inconvenientes y limitaciones. Broncoespasmo, mal funcionamiento del equipo, la aplicación de la oclusión de la punta del tubo de PEEP o cricoides presión puede resultar en falta de detección de CO2.

Con la intubación esofágica, pequeñas formas de onda se pueden ver de forma transitoria como consecuencia de $\mathrm{CO} 2$ que ha entrado en el estómago durante la ventilación con mascarilla o de las bebidas carbonatadas o medicamentos. Esto podría dar la impresión de que el tubo está colocado correctamente en la tráquea. Sin embargo, rápida disminución de concentración y anormales formas de onda por lo general diferenciar esofágico de intubación traqueal.

El CO2End-tidalpuede ayudar en la realización de una intubación oral o nasal ciego. Un capnógrafo se une al tubo traqueal a medida que se inserta en un paciente con respiración espontánea y el CO2 forma de onda y o pico de nivel de CO2 utilizado como una guía. A medida que el tubo se aproxima a la laringe, el CO2aumenta y disminuye a medida que se aleja. A medida que el tubo se hace avanzar y la punta pasa entre las cuerdas vocales, se observa un capnograma normal. La capnografía se puede utilizar para identificar colocación de la aguja durante cricotirotomía transtraqueal. La capnografía puede ser utilizado en conjunción con un introductor de estilete de chorro en los casos en que la apertura de la glotis no puede ser visualizada (Dorsch, 1999).

La capnografía también se ha utilizado para verificar la intubación traqueal durante la broncoscopia de fibra óptica despierto uniendo el capnógrafo al conector de aspiración del ámbito de aplicación. Esto es especialmente útil cuando la visualización de fibra óptica es obstaculizada (Wolf \& Gravenstein, 2007) 


\section{Alteraciones endocrinólogas y metabólicas de las enfermedades criticas}

Vol. 3, núm. 2., (2019)

Carlos Bruno Guerrero Cedeño; César Antonio Moran Vargas; Gabriela Patricia Bajaña Aguilar

La capnografía se utiliza para ayudar en la determinación de la posición correcta y detectar el desprendimiento de un tubo de doble lumen bronquial. El examen de la forma de onda de cada pulmón durante la sujeción y el desamarre procedimientos puede verificar el posicionamiento correcto.

Un monitor de $\mathrm{CO} 2$ se utiliza para controlar la frecuencia respiratoria de $\mathrm{CO}$ 2exhalado en pacientes no intubados con respiración espontánea. Apnea u obstrucción de las vías pueden ser detectados. Si la ventilación del espacio de la respiración es la nueva respiración inadecuada se producirá y puede ser detectado por un aumento del nivel de CO2inspirada.Pico en los niveles de $\mathrm{CO} 2$ niveles se correlacionan más estrechamente con $\mathrm{Pa} \mathrm{CO} 2$ que los niveles de marea de gama media. correlación pobre se asocia con obstrucción de las vías respiratorias parcialy las altas tasas respiratorias. Los resultados pueden ser mejorados por aislar insuflado $\mathrm{O} 2$ de los gases exhalados, observando la forma de onda para la configuración normal, y la disminución de latasa de flujo O2. En respiracion por la boca, la cánula puede ser realineada sobre la boca o la boca puede estar cerrada.

Otro uso importante de la capnografía ha sido determinar las necesidades de ventilación correctos (ventilación minuto) durante la ventilación controlada. Este uso se extiende fácilmente a la monitorización continua de la ventilación espontánea y a la monitorización de la ventilación adecuada durante la titulación de agentes anestésicos que deprimen la ventilación. En los circuitos de nueva respiración parcial y en la anestesia de bajo flujo, capnografía facilita el ajuste de flujo de gas fresco, que es un determinante importante de los niveles CO2, ya que puede aumentar la ventilación minuto. La forma de un capnograma reinspiración parcial puede variar mucho, dependiendo de la frecuencia ventilatoria y el volumen tidal. 


\section{Importancia de la Capnografía durante el monitoreo anestésico}

Vol. 3, núm. 2., (2019)

Carlos Bruno Guerrero Cedeño; César Antonio Moran Vargas; Gabriela Patricia Bajaña Aguilar

Los pacientes que están parcialmente paralizado con relajantes musculares pueden hacer esfuerzos respiratorios. Esto puede alertar al anestesista que el relajante muscular se está desvaneciendo. Puede ocurrir en cualquier parte del ciclo respiratorio (Figura 7). Si la depresión se produce en el tercio posterior de la forma de onda que se conoce como curare hendidura (Fig.8). La capnografía también puede ayudar mientras destete (Fig. 9).

Figura 7: esfuerzos respiratorios espontáneos durante la ventilación mecánica.

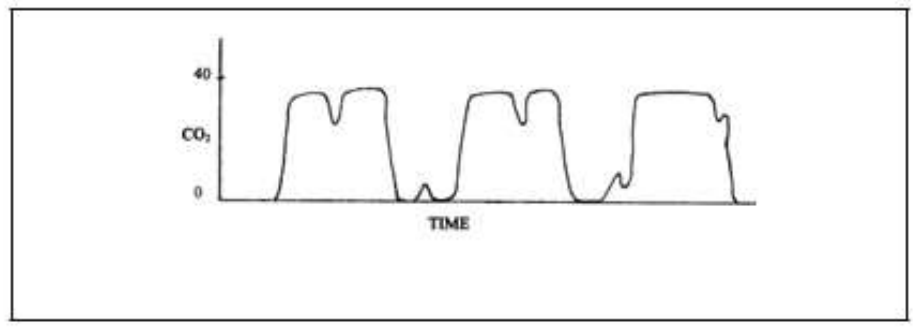

Figura 8: El curare hendidura o muesca

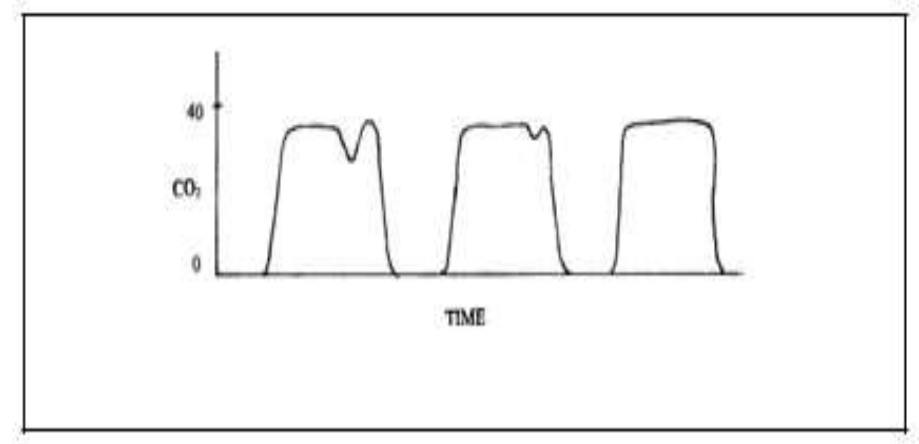




\section{Alteraciones endocrinólogas y metabólicas de las enfermedades criticas}

Vol. 3, núm. 2., (2019)

Carlos Bruno Guerrero Cedeño; César Antonio Moran Vargas; Gabriela Patricia Bajaña Aguilar

Figura 9: Volver a la ventilación espontánea

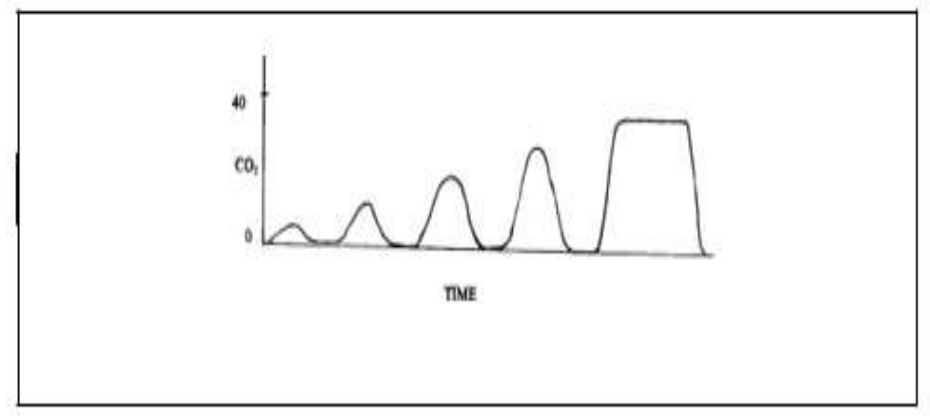

Monitoreo de PET CO2 puede ser utilizado con éxito para determinar los niveles de CO2 Pa durante alta frecuencia de ventilación de chorro (ARMAFJ) mediante la entrega de un solo aliento de gran volumen corriente y la medición de PET CO2 durante una breve interrupción de HFJV. (Capnography, s.f.)

Aparatos de anestesia

Fugas del circuito (Fig.10), que disminuyen el volumen minuto, son detectados por un aumento gradual de CO2 PET. monitores de CO2 detectan la desconexión instantánea en pacientes paralizados. monitorización de $\mathrm{CO} 2$ da una advertencia temprana de retención de $\mathrm{CO} 2$ por el paciente debido a un sistema anestésico defectuoso o mal funcionamiento de las válvulas en los sistemas de círculo anestésicos. 


\section{Importancia de la Capnografía durante el monitoreo anestésico}

Vol. 3, núm. 2., (2019)

Carlos Bruno Guerrero Cedeño; César Antonio Moran Vargas; Gabriela Patricia Bajaña Aguilar

Figura 10: Una fuga en la línea de muestreo durante la ventilación con presión positiva

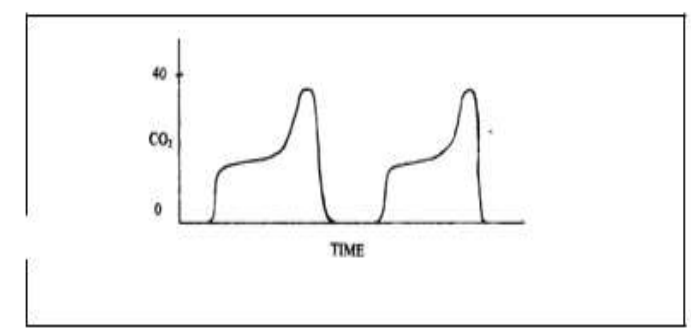

Una oclusión total o extubación accidental de los resultados de tubo endotraqueal en una disminución abrupta en PET CO2. La capnografía es más valioso que capnometría en la detección de los tubos endotraqueales parcialmente estrangulada, como las distorsiones en $\mathrm{CO} 2$ formas de onda (fase prolongada II, más pronunciada de fase III, la altura irregular de la $\mathrm{CO} 2$ formas de onda) se producen antes que los cambios en PET CO 2. Sin embargo, debe haber al menos 50\% de oclusión del tubo endotraqueal para producir cambios en PET CO2 o en las formas de ondaCO2.

\section{Capnografía en lactantes y niños pequeños}

El capnómetro corriente principal son más precisos que capnómetro de flujo lateral para la medida de $\mathrm{CO} 2$ sitio proximal de PET pero pueden causar la nueva respiración inaceptable. Distal PET CO2 debe utilizarse si el peso del niño es $<12 \mathrm{~kg}$, y durante el uso de circuitos de nueva respiración parcial.

Recientemente, la tecnología Microstream (NBP-75, NellcorPuritan Bennett, Plesanton, CA, EE.UU.) se ha introducido con un caudal de aspiración de 30mlmin-1. pero con el tiempo de respuesta rápida que quita las desventajas de capnógrafos convencionales. Se proporciona 


\section{Alteraciones endocrinólogas y metabólicas de las enfermedades criticas \\ Vol. 3, núm. 2., (2019) \\ Carlos Bruno Guerrero Cedeño; César Antonio Moran Vargas; Gabriela Patricia Bajaña Aguilar información precisa PET CO2 mediciones, minimiza las distorsiones en formas de ondaCO2 y reduce la posibilidad de secreciones que ingresen al sistema.}

\section{Conclusiones.}

Los resultados de esta revisión sistemática serán valiosos para la clínica práctica e investigación. Esta revisión sistemática demuestra el estado actual de la evidencia sobre los beneficios para la seguridad del paciente que pueden estar asociados con el uso de capnografía durante el PSA durante los procedimientos clínicos dentro de las instalaciones hospitalarias. Reduciendo el riesgo dela mayoría de evento con antecedente común (hipoxia por oxigenación inadecuada o ventilación) para muerte relacionada con sedación y los déficits neurológicos permanente serían un fuerte indicador y es probable que la monitorización de la capnografía mejore la seguridad al paciente durante el PSA. Una síntesis de la evidencia existente ayudará a los médicos a integrar los hallazgos en sus prácticas.

\section{Bibliografía.}

Adams, A. (1989). Capnography and pulse oximetry. Recent Advances in Anaesthesia and Analgesia, 155-175.

Bolívar, J. (2015). Investigación Documental. México. Pax.

Capnography. (s.f.). Obtenido de www.capnography.com

Castro, J. (2016). Técnicas Documentales. México. Limusa.

Davila, A. (2015). Concepto de terminos cientificos.Caracas: Oasis.

Dorsch, J. (1999). Understanding Anaesthesia Equipment, Gas monitoring. 679-753.

Kalenda, Z. (2009). Mastering Infrared Capnograhy. The Netherlands: Kerckebosch- Zeist. 


\section{Importancia de la Capnografía durante el monitoreo anestésico}

Vol. 3, núm. 2., (2019)

Carlos Bruno Guerrero Cedeño; César Antonio Moran Vargas; Gabriela Patricia Bajaña Aguilar

Mollgaard, K. (2009). Acoustic gas measurement. Biomedical instrumentation and Technology, 495-497.

Raemer, D., \& Philip, J. (1990). Monitoring in Anesthesia and Critical Care Medicine. 2nd edition. New York: Churchill Livingstone.

Website of the Indian Society of Anaesthesiologists. (2000). Obtenido de www.isa-india.org/.

Wolf, L., \& Gravenstein, D. (2007). Capnography during fiberoptic bronchoscopy to verify tracheal intubation. Anesth Analg, 701-702.

$$
\text { (9) (1) }(0)
$$

RECONOCIMIENTO-NOCOMERCIAL-COMPARTIRIGUAL

CC BY-NC-SA

ESTA LICENCIA PERMITE A OTROS ENTREMEZCLAR, AJUSTAR Y CONSTRUIR A PARTIR DE SU OBRA CON FINES NO

COMERCIALES, SIEMPRE Y CUANDO LE RECONOZCAN LA AUTORÍA Y SUS NUEVAS CREACIONES ESTÉN BAJO UNA LICENCIA CON LOS MISMOS TÉRMINOS. 